\title{
Fatty Liver Characterization and Classification by Ultrasound
}

\author{
Ricardo Ribeiro $^{1,2, \star}$ and João Sanches ${ }^{1,3, \star \star}$ \\ 1 Institute for Systems and Robotics (ISR) \\ 2 Escola Superior de Tecnologia da Saúde de Lisboa (ESTeSL) \\ ${ }^{3}$ Instituto Superior Técnico (IST) \\ Lisbon, Portugal \\ ricardo.ribeiro@estesl.ipl.pt
}

\begin{abstract}
Steatosis, also known as fatty liver, corresponds to an abnormal retention of lipids within the hepatic cells and reflects an impairment of the normal processes of synthesis and elimination of fat. Several causes may lead to this condition, namely obesity, diabetes, or alcoholism.

In this paper an automatic classification algorithm is proposed for the diagnosis of the liver steatosis from ultrasound images. The features are selected in order to catch the same characteristics used by the physicians in the diagnosis of the disease based on visual inspection of the ultrasound images.

The algorithm, designed in a Bayesian framework, computes two images: i)a despeckled one, containing the anatomic and echogenic information of the liver, and ii) an image containing only the speckle used to compute the textural features. These images are computed from the estimated RF signal generated by the ultrasound probe where the dynamic range compression performed by the equipment is taken into account.

A Bayes classifier, trained with data manually classified by expert clinicians and used as ground truth, reaches an overall accuracy of $95 \%$ and a $100 \%$ of sensitivity.

The main novelties of the method are the estimations of the RF and speckle images which make it possible to accurately compute textural features of the liver parenchyma relevant for the diagnosis.
\end{abstract}

Keywords: Ultrasound, Speckle, Bayesian, Steatosis Diagnosis.

\section{Introduction}

Fatty infiltration of the liver (steatosis), occurs when the fat content of the hepatocytes increases [12]. Patients with fatty liver are usually symptom free and the disease is typically detected by chance [13]. It is estimate that the prevalence of this disease in the United States and Europe ranges from $14-20 \%$ and it is related directly with the obesity, diabetes, or alcoholism [3].

Liver biopsy is the more accurate method to diagnose a fatty liver. However, since it is invasive it is only used when the other non invasive methods fail. Within

\footnotetext{
* Corresponding author.

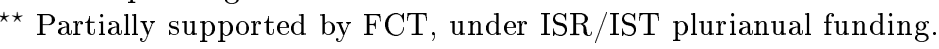


the non invasive methods, the imaging methods as ultrasound (US), computarized tomography $(\mathrm{CT})$ and nuclear magnetic resonance (MRI) plays a great part in diagnosing and quantifying fatty liver [4. Diagnosis based on ultrasound images is, among the non invasive methods, the preferred one because it is non-ionizing, non-invasive and is available in most of the medical and clinical facilities.

In general, the diffuse liver diseases appear in the US images with increased echogenicity of the parenchyma and some times, in the pre-cirrhotic stages, with textural changes [2. In the case of the fatty liver this effect are accompanied by an acoustic penetration decreasing and a reduction on the blood vessels and diaphragm definition [5. However, a simple human visual inspection of the images is not enough to get an accurate diagnosis of the disease stage [6] and highly experimented operators are needed to detect subtle changes on the hepatic texture [5]. In fact, the criteria used to assess this disease by visual inspection are not well defined and the diagnosis is in general highly subjective and operatordependent [7 (Fig:1). Additionally, the poor quality of the images, the speckle that corrupt them and differences on the tunning parameters of the US scanner prevent the adoption of an unified standard diagnosis procedure [8].
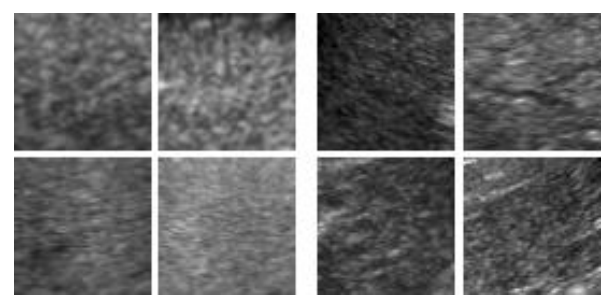

Fig. 1. Liver tissues samples: Steatosis (two left columns) and Normal (two right columns)

Quantitative tissue characterization technique (QTCT) [7], could increase the usefulness of US for the evaluation of diffuse liver disease. QTCT is based on extraction of features from the US images for classification and identification purposes and therefore for diagnosis purposes. The most common features described in the literature are based on the first order statistics $[6|5| 7$, , co-occurrence matrix [79, wavelet transform [109], attenuation and backscattering parameters 7 and backscattering coefficient [7. [11] proposes a tissue characterization from US images of the thyroid based on features extracted from the Radon transform in order to discriminate pattern directionality characteristics.

In this paper a classifier is proposed for automatic diagnosis of the steatotic disease from ultrasound images of the liver parenchyma. The algorithm is based on the usual criteria used by the physicians in the diagnostic process through visual inspection of the ultrasound images (see Fig. 1). The method uses a Bayes classifier that combines intensity features extracted from the estimated despeckled image and textural features extracted from the estimated speckle image after compensation of the log compression performed by the ultrasound equipment. 


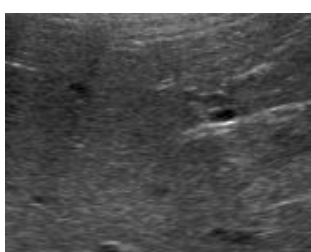

(a) US

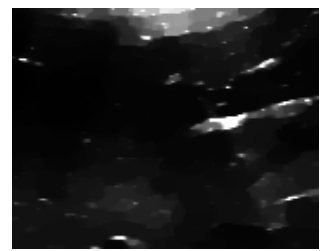

(c) Despeckled

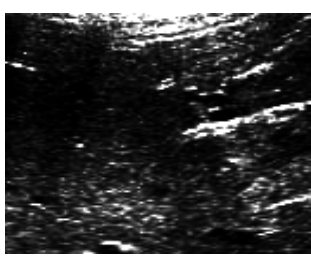

(b) RF

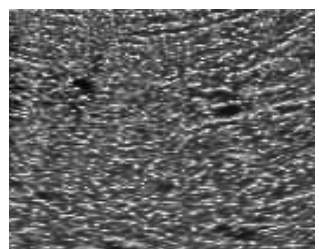

(d) Speckle

Fig. 2. Original ultrasound image a), the estimated RF signal b) and the two images from which intensity c) and texture d) features are extracted

The paper is organized as follows: section 2 formulates the problem and characterize the data. In section 3 the classification algorithm is described and in section 4 examples using real data are shown. Section 5 concludes the paper.

\section{Problem Formulation}

In this paper an objective method is proposed where the observed ultrasound images are pre-processed and the original RF signal generated by the ultrasound probe is estimated, as shown in Fig, 2 b). The RF image is used to estimate two images: a despeckled one (see Fig.2 c)) containing the anatomic details from which intensity features are extracted and a second image containing only the speckle (see Fig 2.2d) from which the textural information is obtained. The estimation of the RF signal makes it possible to attenuate the dependence on the the specific tunning parameters of the scanner, such as brightness and contrast, and therefore to reduce the subjective nature of the traditional diagnosis based on visual inspection.

The estimation of the RF and of despeckled anatomic images is performed using the Bayesian methods proposed in [12] and [13] respectively. In these methods the compression operation performed by the ultrasound scanner is modeled by the following logarithmic function

$$
\mathbf{Z}=a \log (\mathbf{Y}+1)+b
$$

where $\mathbf{Z}$ is the observed US image and $\mathbf{Y}$ is the RF image to be estimated. The $\mathrm{RF}$ image, corrupted by speckle, is described by a Rayleigh distribution 


$$
p\left(y_{i}\right)=\frac{y_{i}}{f_{i}} e^{-\frac{y_{i}}{2 f_{i}}}
$$

where $\mathbf{F}=\left\{f_{i}\right\}$ is the despeckled image and $f_{i}$ and $y_{i}$ are the $i^{\text {th }}$ despeckled and speckle pixels respectively.

In this paper the estimated noise field is used to extract the textural features needed for the automatic diagnosis of the steatosis. The speckle corrupting the ultrasound images is multiplicative in the sense that its variance depends on the underlying signal $\mathbf{F}$. The image formation model may be formulated as follows:

$$
y=\eta \sqrt{f}
$$

where $f$ is a pixel intensity of the despeckled image and $\eta$ is the corresponding noisy intensity. In this model, the noise field, $\eta$, is independent of the signal as occurs in the common additive white Gaussian noise (AWGN) model where the noisy pixels, $y=f+\eta$, are the sum of two independent terms, $f$ and $\eta$. In the case of the multiplicative noise the corruption operation is not additive but multiplicative as shown in (3). The distribution of $\eta$ is

$$
p(\eta)=\left|\frac{d y}{d \eta}\right| p(y)=\eta e^{-\eta^{2} / 2}, \quad \eta \geq 0
$$

which is a unit parameter Rayleigh distribution independent of $f$.

\section{Classifier}

Visual classification of diffuse fatty infiltration disease from US images is usually based on two main features: i) increase in liver parenchyma echogenicity and ii) decreasing on the acoustic penetration with a corresponding visualization loss of the diaphragm and hepatic vessels [2]. In this condition the pixel intensities strongly decay with image depth ( $y$ axis).

The feature associated with the intensity decay is obtained by a linear regression computed over the mean values of each line, $h(i)=\frac{1}{M} \sum_{j=1}^{M} f_{i, j}$ of the despeckled image $\mathbf{F}$ where the following cost function is minimized

$$
J=\sum_{i=0}^{N}(m i+b-h(i))^{2}
$$

with $N$ and $M$ being the number of lines and columns respectively and $m$ the slope that is used to quantify the depth decay as displayed in Fig 3 ,

The textural features are obtained from the speckle image, $\eta_{i, j}=y_{i, j} / \sqrt{f_{i, j}}$ by computing its first Haar wavelet decomposition vertical and horizontal details. The energies of these two images, $E d V$ and $E d H$ respectively, are used as textural discriminant features of healthy and steatotic livers.

A Bayes classifier based on these three features, $\mathbf{x}=\{m, E d V, E d H\}$, is trained with data classified by expert clinicians in two classes, Normal, $\omega_{N}$, 

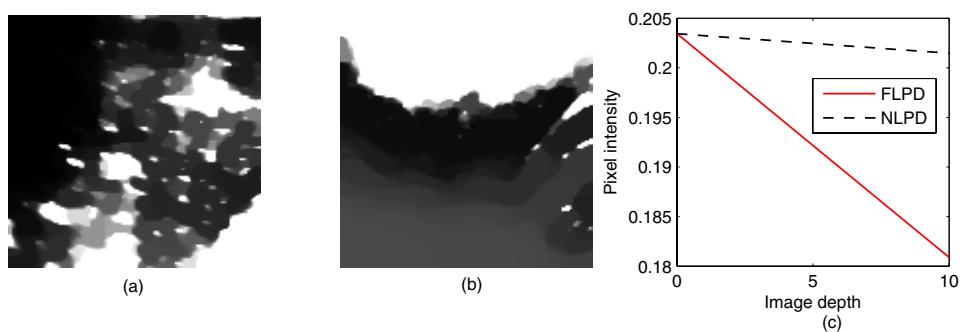

Fig. 3. a) despeckled ultrasound image of a normal liver, b) despeckled ultrasound image of a fatty liver and the c) represents two straight lines with slopes obtained by averaging the $m$ slopes of each set, normal (NLPD) and fatty livers (FLPD), obtained from (5)

and Fatty, $\omega_{F}$. It is assumed that the vector of features are multivariate normal distributed [109] with different means, $\left\{\mu_{N}, \mu_{F}\right\}$ and covariance, $\left\{\Sigma_{N}, \Sigma_{F}\right\}$, matrices. The linear discriminant functions are

$$
g_{\tau}(\mathbf{x})=-\frac{1}{2}\left(\mathbf{x}-\mu_{\tau}\right)^{T} \Sigma_{\tau}\left(\mathbf{x}-\mu_{\tau}\right)-\frac{1}{2} \log \left|\Sigma_{\tau}\right|+\log P\left(w_{\tau}\right)
$$

where $\tau \in\{N, F\}$. The classification of a given vector of features $\mathbf{x}$ is performed according with

$$
\begin{cases}\text { Fatty } & \text { if } g_{F}(\mathbf{x})>g_{N}(\mathbf{x}) \\ \text { Normal } & \text { otherwise }\end{cases}
$$

\section{Experimental Results}

The US images used in this paper were obtained by a common US equipment in a hospital facility and all images were stored in DICOM format. The US equipment settings were not standardize since we estimate the original RF signal and despeckled image.

Two sets of images were stored: normal hepatic parenchyma (10 images) from 5 subjects and fatty hepatic parenchyma (10 images) from 5 subjects. The classification was made manually by the operators and confirmed by indicators obtained from laboratorial analysis.

The training process of the recognition system is operated in two modes: training (learning) and classification (testing) [14]. For each class, $w_{1}=$ fatty and $w_{2}=$ normal, the textural features, mean and covariance, were estimated using 10 images with the leave-one-out cross-validation method.

Cross-validation is often used to estimate the generalization ability of a statistical classifier [15]. This method was used due to the lack of enough data for the training and testing procedures. 
The classification accuracy were obtained by

$$
C a=1-(\text { false-negative rate }+ \text { false-positive } \text { rate }),
$$

where the false-negative rate is defined as the probability that the classification result indicates a normal liver while the true diagnosis is indeed a liver disease and the false-positive is defined as the probability that the classification result indicates a liver disease while the true diagnosis is indeed a normal liver.

Complementary laboratory analysis of the fatty livers are nonspecific but include modest elevations of the aspartate aminotransferase (AST), alanine aminotransferase (ALT), and -glutamyl transpeptidase (GGTP), accompanied by hypertriglyceridemia, hypercholesterolemia 3 .

From each image, one ROI of 128 by 128 pixels along the center line have been selected.

The overall accuracy of the Bayesian classifier using the proposed algorithm and combining the features selected was $95 \%$, which is a promising result. In this type of studies one of the most important characteristics is to optimize the sensitivity (conditional probability of detecting a disease while there is in fact a liver disease) and specificity (conditional probability of detecting a normal liver while the liver is indeed normal), in order to reduce the false-negative and the false-positive rate. The false-negative rate, in a first instance, should be completely avoided since it represents a danger to the patient. In this sense, the sensitivity parameter was optimal, since we obtain $100 \%$ in the classification process and in terms of specificity of $95 \%$.

The results illustrate a high sensitivity of detecting this type of disease. Also the algorithm proposed apparently is not depending on the place of scanning and on the operator technique, since the results were not influenced by the presence of blood vessel and bile ducts within the liver parenchyma chosen for each ROI, and also by the changes in US image parameters.

As it can be observed in Fig. 4, the results have shown the usefulness of the features in the detection of pathological cases because almost no overlapping is observed between both statistical clouds. The mean cloud parameters for the healthy livers are: Slope $\left(\mu=0.8014 ; \sigma^{2}=0.0113\right)$; EdH $\left(\mu=0.1997 ; \sigma^{2}=0.0021\right)$; $\operatorname{EdV}\left(\mu=0.0466 ; \sigma^{2}=2.6068 \mathrm{e}-04\right)$ and for the Fatty livers are: Slope $(\mu=0.4796$; $\left.\sigma^{2}=0.0337\right) ; \mathrm{EdH}\left(\mu=0.0979 ; \sigma^{2}=7.1793 \mathrm{e}-04\right) ; \mathrm{EdV}\left(\mu=0.0678 ; \sigma^{2}=2.6477 \mathrm{e}-04\right)$.

As [16] propose, we assume that the physics of US scanning and the speckle is separable into factors arising from orthogonal directions. The $x$ direction will be the transverse (lateral) direction. The $z$ direction will be the range (longitudinal) direction, or the direction of insonification. The physics in the transverse direction is governed by diffraction and interference effects of the coherent pressure (scalar) fields. The physics in the range direction is governed by the shape of the RF pulse that is sent and received. From the analysis of the results obtain by the EdH and EdV it seems that the US images of the liver have different behavior when it is normal or with fatty deposits. Characteristically the normal liver has higher horizontal and lower vertical energy details when compared to the fatty liver. So when the fat content in the liver increases the predominant direction 


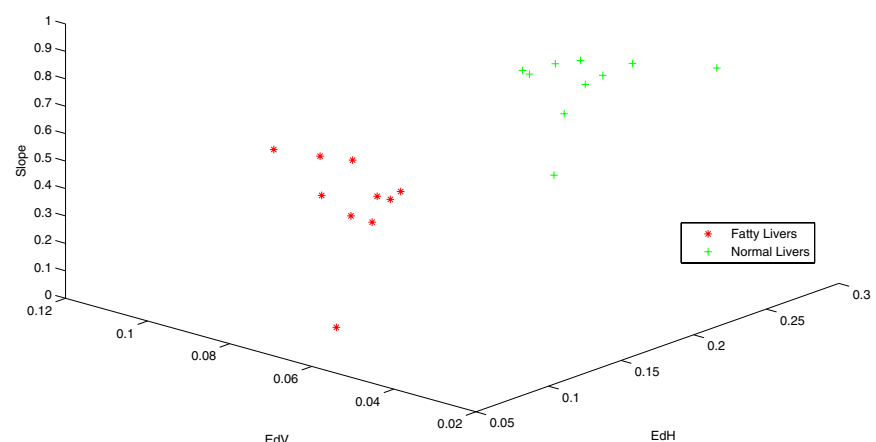

Fig. 4. Distribution of fatty vs normal livers according to the selected features

is $z$, and in normal livers the predominant direction, according to the textural information of the US image, is $x$, which is related with the histologic findings in the case of fatty livers (macrovesicular fat).

\section{Conclusions}

The proposed algorithm, based on the quantification of the visual diagnostic criteria, produces an objective analysis of the liver condition, which could be helpful to aid the diagnosis in this type of diffuse liver disease. The results are very promising in terms of sensitivity and specificity, which could aid in the detection in early stages of this disease, in asymptomatic patients, in order to prevent the progression to major lesions (steato-hepatitis or even cirrhosis). The features used in this paper are mathematical and objective formulations of the criteria used in the subjective and operator dependent traditional procedure based on manual visual inspection.

The RF signal compensation and the separation of the despeckled component from the original one is the key issue for the success of the proposed method.

Further studies in this area are needed. To develop a more robust algorithm, we need to increase the number of patients. Perform the feature extraction methods in the original US images, prior to machine processing. Other classification methods, such as neural network and the support vector machine classifiers should be tested, to optimize the process.

\section{References}

1. Sherlock, S., Dooley, J.: Diseases of the liver and Biliary System, 11th edn. Blackwell Science Ltd., Malden (2002)

2. Droga, V., Rubens, D.: Ultrasound Secrets. Hanley and Belfus (2004)

3. Fauci, A., et al.: Harrison's Principles of Internal Medicine, 17th edn. McGrawHill's, New York (2008) 
4. Lupsor, M., Badea, R., Nedevschi, S., Mitrea, D., Florea, M.: Ultrasonography contribution to hepatic steatosis quantification. possibilities of improving this method through computerized analysis of ultrasonic image. In: IEEE International Conference on Automation, Quality and Testing, Robotics, vol. 2, pp. 478-483 (May 2006)

5. Lee, C.H., et al.: Usefulness of standard deviation on the histogram of ultrasound as a quantitative value for hepatic parenchymal echo texture; preliminary study. Ultrasound in Med. \& Biol. 32, 1817-1826 (2006)

6. Maeda, K., et al.: Quantification of sonographic echogenicity with grey-level histogram width: a clinical tissue characterization. Ultrasound in Med. \& Biol. 24, $225-234$ (1998)

7. Kadah, Y.M., et al.: Classification algorithms for quantitative tissue characterization of diffuse liver disease from ultrasound images. IEEE Trans. Med. Imaging 15, 466-478 (1996)

8. Li, G., et al.: Computer aided diagnosis of fatty liver ultrasonic images based on support vector machine. In: 30th Annual International Conference of the IEEE, Engineering in Medicine and Biology Society (EMBS 2008), pp. 4768-4771 (2008)

9. Yeh, W.-C., et al.: Liver fibrosis grade classification with b-mode ultrasound. Ultrasound in Med. \& Biol. 29, 1229-1235 (2003)

10. Mojsilovic, A., et al.: Characterization of visually diffuse diseases from b-scan liver images using non-separable wavelet transform. IEEE Trans. Med. Imaging 17, 541549 (1998)

11. Savelonas, M.A., et al.: Computational characterization of thyroid tissue in the radon domain. In: Twentieth IEEE International Symposium on Computer-Based Medical Systems (2007)

12. Seabra, J., Sanches, J.: Modeling log-compressed ultrasound images for radio frequency signal recovery. In: 30th Annual International Conference of the IEEE, Engineering in Medicine and Biology Society (EMBS 2008) (2008)

13. Seabra, J., Xavier, J., Sanches, J.: Convex ultrasound image reconstruction with log-euclidean priors. In: 30th Annual International Conference of the IEEE, Engineering in Medicine and Biology Society (EMBS 2008) (2008)

14. Jain, A., et al.: Statical pattern recognition: A review. IEEE Transactions on pattern analysis and machine intelligence $22,4-37$ (2000)

15. Cawley, G., Talbot, N.: Efficient leave-one-out cross-validation of kernel fisher discriminant classifiers. Pattern Recognition 36, 2585-2592 (2003)

16. Wagner, R., et al.: Statistics of speckle in ultrasound b-scans. IEEE Transactions on Sonics and Ultrasonics 30, 156-163 (1983) 